Check for updates

Cite this: RSC Adv., 2018, 8, 7949

\title{
Sweeping-micelle to solvent stacking for the on- line preconcentration and determination of organic acids in Angelica sinensis by capillary electrophoresis $\uparrow$
}

\begin{abstract}
Xiumin Yang, (D) Lin Hao, Shuaihua Zhang, Chun Wang (D) and Zhi Wang*
A novel on-line two-step stacking preconcentration method by sweeping plus micelle to solvent stacking in capillary zone electrophoresis was developed for the simultaneous determination of three organic anions (vanillic acid, ferulic acid and cinnamic acid) in Angelica sinensis. Hexadimethrine bromide was used for electroosmotic flow reversal. The main experimental parameters that affected the separation and sensitivity were investigated and optimized. The best separation was achieved in $50 \mathrm{mM}$ ammonium acetate $(\mathrm{pH}$ 12.0) containing 50\% methanol (v/v) under a negative voltage of $20 \mathrm{kV}$. The micellar solution was a mixed solution comprised of $12 \mathrm{mM}$ cetyltrimethyl ammonium bromide and $20 \mathrm{mM}$ ammonium acetate. After injecting a short plug $(0.5 \mathrm{psi}, 30 \mathrm{~s})$ of micellar solution, the sample was introduced into the capillary at $0.5 \mathrm{psi}$ for $45 \mathrm{~s}$. Under the optimal conditions, the sensitivity enhancement factors obtained by the developed method were between 42 and 77. The intra-day $(n=6)$ and inter-day $(n=5)$ precisions of the method expressed as their relative standard deviations were found to be less than $7.2 \%$. The Angelica sinensis sample was pulverized and then refluxed in 95\% ethanol and filtered. After an aliquot of the extractant was dried, it was reconstituted in $20 \mathrm{mM}$ ammonium acetate for capillary electrophoresis analysis. The recoveries of the analytes by this method for the analysis of Angelica sinensis were in the range of $94.4 \%$ to $108.4 \%$.
\end{abstract}

Received 20th October 2017 Accepted 7th February 2018

DOI: 10.1039/c7ra11553a

rsc.li/rsc-advances
Compared with HPLC and GC, CE has advantages such as short analysis time and high separation efficiency, almost organic solvent-free analysis, and small sample amount requirements. Therefore, CE has become more and more popular for the analysis of the bioactive components in TCMs. Nevertheless, due to the short optical path length across the capillary, the main drawback of CE is its poor detection sensitivity with ultraviolet detection. Therefore, various on-line sample preconcentration or stacking methods prior to $\mathrm{CE}$ have been established (e.g., sweeping, dynamic pH junction, large volume sample stacking (LVSS) transient isotachophoresis and micelle to solvent stacking (MSS)). ${ }^{15,16}$

MSS was first introduced by Quirino in 2009 and relies on the change in the direction of the effective electrophoretic mobility of the charged analytes in the presence of organic solvents and micelles. ${ }^{17}$ It is a relatively new on-line CE preconcentration technique and has been applied for the detection of cations (antipsychotic drugs, $\beta$-blockers, tricyclic antidepressant medicines and alkaloids, as well as herbicides). ${ }^{18}$ Thereafter, when compared with that of MSS alone, further improvements in the detection sensitivity were reported by coupling it with sweeping ${ }^{19-21}$ or field enhanced sample injection ${ }^{22-25}$ or both..$^{26,27}$ In 2011, for anionic analytes, the new two-step stacking by sweeping and MSS using cationic micelles in co-electroosmotic
Department of Chemistry, College of Science, Agricultural University of Hebei, Baoding 071001, China.E-mail:wangzhi@hebau.edu.cn; zhiwang2013@aliyun.com; Fax: +86312-7521513; Tel: +86-312-7521513

$\dagger$ Electronic supplementary information (ESI) available. See DOI: 10.1039/c7ra11553a 
flow (EOF) was developed. ${ }^{28}$ The co-EOF condition where the direction of EOF is the same as the anions was satisfied by a positive dynamic coating of a fused silica capillary using hexadimethrine bromide (HDMB). In such a process, the background solution (BGS) contained organic solvent and the sample solution was devoid of micelles. A cationic micellar solution was injected before the sample solution and a negative voltage was applied. The analytes in the micelle-free sample zone were swept by the micelles brought to the MSS boundary where the second stacking step was induced by the presence of the organic solvent in BGS. However, the study ${ }^{28}$ mainly focused on the stacking mechanism of the proposed method using hypolipidaemic drugs, anti-inflammatory drugs and herbicides as the model analytes, and the enrichment factors for the analytes were only about 20 . So far, the applications of sweepingMSS for other anionic analytes have not been investigated.

In this study, we report a two-step on-line stacking technique, MSS coupled with sweeping, to analyze three anionic organic acids named vanillic acid, ferulic acid and cinnamic acid found in Angelica sinensis. The experimental variables that may affect the strategy are investigated. The developed method is simple, sensitive and suitable for the determination of the organic acids in TCM.

\section{Experimental}

\subsection{Reagents}

Organic standards of vanillic acid, ferulic acid and cinnamic acid (all 98\% pure) were purchased from Tianjin Heowns Biochem Technologies. Sinapic acid (98\%), cetyltrimethyl ammonium bromide (CTAB) and hexadimethrine bromide (HDMB) (94\%) were purchased from Sigma-Aldrich (St. Louis, MO, USA). Angelica sinensis was obtained from Yubaotang Pharmacy (Baoding, China). Ammonium acetate, sodium hydroxide, hydrochloric acid, boracic acid and HPLC-grade methanol were purchased from Kaitong Chemical Reagent Co., Ltd. (Tianjin, China). All reagents were of analytical grade and used without further purification. All the solvents were filtered through a 0.45 $\mu \mathrm{m}$ Micro Science membrane filter (Tianjin Automatic Science Instrument Co., Ltd. Tianjin, China). The water used throughout the study was double-distilled using an SZ-93 automatic double-distiller (Shanghai Yarong Biochemistry Instrumental Factory, Shanghai, China).

A mixed stock solution containing vanillic acid, ferulic acid and cinnamic acid ( $50 \mu \mathrm{g} \mathrm{mL^{-1 }}$ each), and stock solutions of the internal standard (IS) sinapic acid at $50 \mu \mathrm{g} \mathrm{mL} \mathrm{m}^{-1}$ and $1.0 \mathrm{mg}$ $\mathrm{mL}^{-1}$ were prepared in absolute ethanol and stored in a refrigerator at $4{ }^{\circ} \mathrm{C}$. A series of standard solutions were prepared by mixing an appropriate amount of the stock solutions with $20 \mathrm{mM}$ ammonium acetate after the stock solution was dried under a gentle stream of nitrogen at room temperature. The BGS was $50 \mathrm{mM}$ ammonium acetate $(\mathrm{pH} 12.0)$ containing $50 \%$ methanol $(\mathrm{v} / \mathrm{v})$, and the micellar solution was $20 \mathrm{mM}$ ammonium acetate containing $12 \mathrm{mM}$ CTAB. Both BGS and the micellar solution were freshly prepared daily and sonicated for $10 \mathrm{~min}$ prior to use.

\subsection{Apparatus}

All CE experiments were performed on a Beckman P/ACE MDQ Capillary Electrophoresis System (Fullerton, CA, USA) equipped with an auto sampler and a diode array detector. An uncoated fused-silica capillary (Yongnian Ruifeng Optical Fiber Factory, Hebei, China) of $50 \mathrm{~cm}$ (effective length, $41.5 \mathrm{~cm}) \times 75 \mu \mathrm{m}$ i.d. was used throughout the experiments. Data acquisition and instrument control were carried out with Beckman P/ACE MDQ 32 Karat software. A PHS-3C pH meter (Hangzhou Dongxing Instrument Factory, Hangzhou, China) was used for the $\mathrm{pH}$ measurements. Conductivity measurements were made using a conductivity meter purchased from Mettler-Toledo instruments (Shanghai) Co., Ltd.

\subsection{Preparation of the samples}

The dried roots of the Angelica sinensis sample were pulverized. $400 \mathrm{mg}$ of Angelica sinensis powder was weighed and $500 \mu \mathrm{L}$ of IS stock solution $\left(1.0 \mathrm{mg} \mathrm{mL}^{-1}\right)$ was added. Next, the powder was refluxed in $20 \mathrm{~mL}$ of $95 \%$ ethanol for $30 \mathrm{~min}$ and then filtered. The residue was refluxed and filtered one more time as described above. Then, all the solvent extracts were combined and transferred into a $50 \mathrm{~mL}$ volumetric flask and made to the mark with 95\% ethanol. $200 \mu \mathrm{L}$ of the resulting solution of the Angelica sinensis sample was evaporated to dryness under a gentle stream of nitrogen at room temperature. Then, the sample solution was obtained by dissolving the residues in $200 \mu \mathrm{L}$ of $20 \mathrm{mM}$ ammonium acetate. The sample solution was filtered through a 0.45 $\mu \mathrm{m}$ syringe filter prior to the $\mathrm{CE}$ experiments.

\subsection{General CE procedures}

Before its first use, the new capillary was sequentially flushed with methanol (10 $\mathrm{min})$, water $(5 \mathrm{~min}), 0.1 \mathrm{M}$ sodium hydroxide (10 $\mathrm{min})$, water (5 min) and 1\% HDMB (60 min) at $20 \mathrm{psi}$. At the start of each day, the capillary was flushed with 1\% HDMB (10 $\mathrm{min}$ ), water ( $5 \mathrm{~min}$ ) followed by BGS ( $5 \mathrm{~min})$. Between runs, the capillary was flushed in sequence with $1 \%$ HDMB (3 min), water (3 min) and BGS (3 $\mathrm{min})$.

The optimized experimental conditions for CE with stacking were as follows: after preconditioning the capillary with BGS, the micellar solution was introduced at 0.5 psi for $30 \mathrm{~s}$. Then, the sample solution prepared in $20 \mathrm{mM}$ ammonium acetate was injected at $0.5 \mathrm{psi}$ for $45 \mathrm{~s}$. The temperature was set at $25^{\circ} \mathrm{C}$ and a negative voltage of $20 \mathrm{kV}$ was applied throughout this procedure. The detection wavelength for the three analytes was set at $215 \mathrm{~nm}$.

\subsection{Determination of the sensitivity enhancement factor}

The focusing efficiency of the current method was assessed by a comparison of its performance under the optimum conditions with that of normal capillary zone electrophoresis (CZE). The sensitivity enhancement factor (SEF) in terms of the peak height was obtained by simply determining the ratio of the peak height obtained by the current method to that obtained by normal CZE injection and then multiplying by the concentration dilution factor. $^{29}$ 


\section{Results and discussion}

\subsection{Optimization of the stacking conditions}

In our study, the concentration of both ammonium acetate and methanol in BGS, the $\mathrm{pH}$ value of BGS, the concentration of both CTAB and ammonium acetate in the micellar solution, and the injection time for both the micellar solution and sample solution were investigated and optimized. A mixed solution of the organic acids and IS $\left(5.0 \mu \mathrm{g} \mathrm{mL}^{-1}\right.$ each) was used for the optimization.

3.1.1. The effect of the concentration of methanol in BGS. The content of organic solvent in BGS is a crucial parameter affecting both the resolution and enrichment of the analytes. Organic solvent can change the electrical conductivity of BGS and weaken the interactions between the analytes and micelles. Methanol is commonly used in MSS. ${ }^{18}$ In this study, the effect of the methanol concentration in BGS was investigated in the range of $40-60 \%$, whereas the ammonium acetate concentration was kept constant at $50 \mathrm{mM}$. As shown in Fig. 1, when the concentration of methanol was $40 \%$, the peaks of the analytes were observed to be smaller than those observed using $50 \%$ or $60 \%$ methanol. The reason for this was ascribed to the low percentage of methanol in BGS being insufficient to lower $k$ at MSSB. On the other hand, when the concentration of methanol was up to $60 \%$, the peaks of the analytes became broad due to low EOF caused by the high percentage of methanol and the IS not being stacked efficiently, which caused the peak shape to be seriously deteriorated. As a result, $50 \%(\mathrm{v} / \mathrm{v})$ methanol in BGS was chosen.

3.1.2. The effect of the concentration of ammonium acetate in BGS. The concentration of the electrolyte in BGS has a significant effect on the resolution. EOF can be reduced with

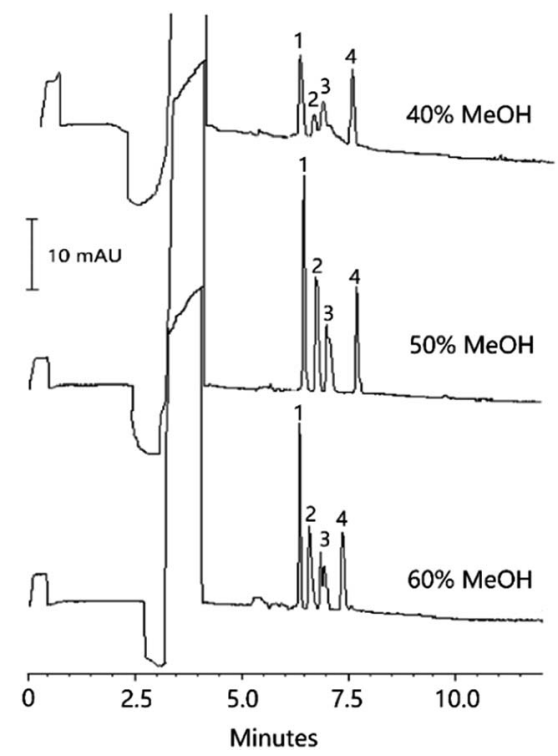

Fig. 1 The effect of the methanol concentration in BGS. Analyte concentration: $5 \mu \mathrm{g} \mathrm{mL}^{-1}$ of the organic acids and IS each. The other conditions are the optimum conditions. Peak identification: 1-vanillic acid, 2-ferulic acid, 3-IS and 4-cinnamic acid. an increase in the electrolyte concentration in BGS; the migration time of the organic acids will be increased and the resolution will be improved. On the other hand, a higher electrolyte concentration can also produce more Joule heat, which can cause an increase in the baseline noise. Among several kinds of commonly used electrolytes, ammonium acetate behaved the best. Therefore, ammonium acetate was chosen for further study. To obtain the best separation and stacking effect for the analytes, the influence of the ammonium acetate concentration was investigated in the range of 30 to $70 \mathrm{mM}$ (Fig. S1A in the ESI $\dagger)$. The results showed that when the concentration of ammonium acetate was changed from 30 to $50 \mathrm{mM}$, the peak heights of the three analytes increased with the increasing concentration of ammonium acetate. However, when the concentration of ammonium acetate was changed from 50 to $70 \mathrm{mM}$, the peak height for vanillic acid decreased slightly, and the stacking efficiency for cinnamic acid first increased slightly and then decreased. Giving an overall consideration, $50 \mathrm{mM}$ ammonium acetate in the BGS was selected for our further studies.

Ohm's law plot was used for the determination of the maximum voltage that can be utilized for BGS. Fig. S2† indicates that when the voltage was between $-5 \mathrm{kV}$ and $-20 \mathrm{kV}$, there was a linear relationship between the applied voltage and the produced current; when the voltage was between $-20 \mathrm{kV}$ and $-25 \mathrm{kV}$, the produced current became much higher than that predicated by the Ohm's law. Hence, $-20 \mathrm{kV}$ was selected as separation voltage.

3.1.3. The effect of the BGS $\mathbf{p H}$. The $\mathrm{pH}$ value of BGS has a significant influence on EOF and therefore, it influences the migration time and separation efficiencies of analytes. In this study, four different $\mathrm{pH}$ values $(11.0,11.5,12.0$ and 12.5) were investigated. Fig. 2 shows that both the peak shapes and resolutions for the analytes and IS were the best at $\mathrm{pH}$ 12.0. When the $\mathrm{pH}$ was lower than 12.0, the stacking efficiency became worse and IS gave two peaks; when the $\mathrm{pH}$ was 12.5 , both the separation efficiency and the resolution between the IS and ferulic acid slightly decreased (see Table S1†). Moreover, when the $\mathrm{pH}$ was 12.5 , the electric current was much higher than that at $\mathrm{pH}$ 12.0. With the increase in current, Joule heat production increased, which resulted in an increase in the baseline noise. Finally, a pH of 12.0 was selected.

3.1.4. The effect of micellar solution and sample solution. Since the charge of the analyte must be opposite to that of the micelle in MSS, the commonly used cationic surfactant CTAB was chosen for stacking. In the MSS process, the concentration of CTAB should be higher than its critical micelle concentration (1.3 $\mathrm{mM})$ for forming micelles. However, its concentration should not be too high, otherwise, the affinity between the analytes and micelles will be too strong to reverse the effective electrophoretic mobility of the analytes at MSSB, which will result in the failure of MSS. To explore the effect of the CTAB concentration in the micellar solution on the focusing efficiency of the analytes, different concentrations of CTAB from 2 to $25 \mathrm{mM}$ were evaluated (Fig. S1B $\dagger$ ). The results showed that the peak heights for all the three analytes increased as the concentration of CTAB was increased from 2.0 to $8 \mathrm{mM}$; when 


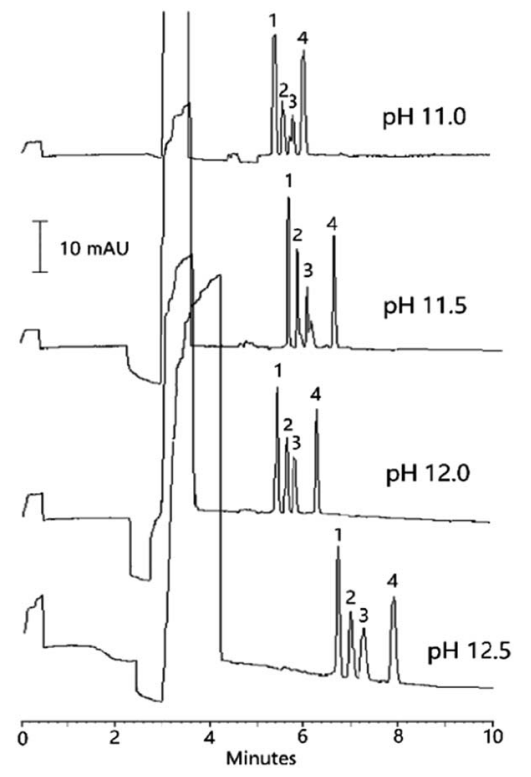

Fig. 2 The effect of the BGS pH. Analyte concentration: $5 \mu \mathrm{g} \mathrm{mL}^{-1}$ of the organic acids and IS each. The other conditions are the optimum conditions. Peak identification: 1-vanillic acid, 2-ferulic acid, 3-IS and 4-cinnamic acid.

the concentration of CTAB was further increased from 8 to $16 \mathrm{mM}$, the peak heights remained almost unchanged and after that, the peak heights decreased sharply from 16 to $25 \mathrm{mM}$. Based on the above results, $12 \mathrm{mM} \mathrm{CTAB}$ was chosen for our further studies.

The conductivity of the micellar solution and sample matrix should be lower than or approximately equal to that of BGS. ${ }^{17}$ In this study, when the concentration of CTAB was kept at $12 \mathrm{mM}$, five different ammonium acetate concentrations (0, 20, 40, 80 and $120 \mathrm{mM}$ ) in the micellar solution were investigated (Fig. S1C $\dagger$ ), and their corresponding micellar solution conductivities were $0.338,2.39,4.16,7.62$ and $11.76 \mathrm{mS} \mathrm{cm}^{-1}$, respectively. As a result, the best separation and focusing efficiency was obtained when $20 \mathrm{mM}$ ammonium acetate was used. Under this condition, the conductivity of the micellar solution $\left(\mathrm{NH}_{4} \mathrm{Ac}\right.$ $20 \mathrm{mM}$, СTAB $12 \mathrm{mM})$ was close to that of BGS $\left(2.72 \mathrm{mS} \mathrm{cm}^{-1}\right)$. The five different ammonium acetate concentrations $(5,10,20$, 30 and $40 \mathrm{mM}$ ) in the sample matrix were also investigated, and their corresponding sample matrix conductivities were 0.543 , $1.06,2.05,3.02$ and $3.82 \mathrm{mS} \mathrm{cm}^{-1}$, respectively. It can be seen from Fig. S1D $\dagger$ that the peak heights of all the three organic acids increased when the ammonium acetate concentration was increased from 5 to $20 \mathrm{mM}$. Then, when the ammonium acetate concentration was further increased from 20 to $40 \mathrm{mM}$, the peak heights remained constant for ferulic acid, slightly increased for cinnamic acid and slightly decreased for vanillic acid. On the basis of the above results, $20 \mathrm{mM}$ ammonium acetate was selected as the sample matrix.

3.1.5. The effect of the injection time. While the injection time for the sample solution was maintained at $45 \mathrm{~s}(0.5 \mathrm{psi})$, the injection time for the micellar solution was varied from 10 to $40 \mathrm{~s}$ (Fig. 3A). When the injection time of the micellar solution was shorter than $20 \mathrm{~s}$, vanillic acid was not stacked completely, demonstrating that the micellar solution was not sufficient. The peak heights for all the three analytes were the highest at $30 \mathrm{~s}$. At an injection time of $40 \mathrm{~s}$, both the resolution and separation efficiency were decreased (Table S2 $\dagger$ ).

When the injection time for the micellar solution was maintained at $0.5 \mathrm{psi}$ for $30 \mathrm{~s}$, the sample injection time in the

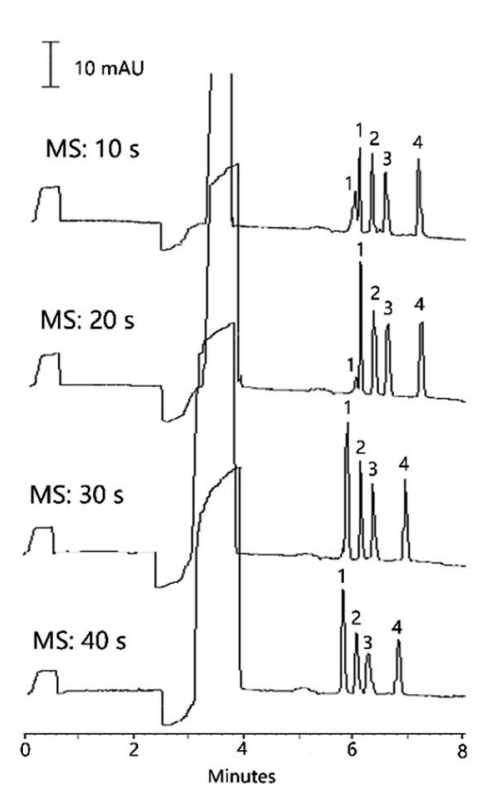

(A)

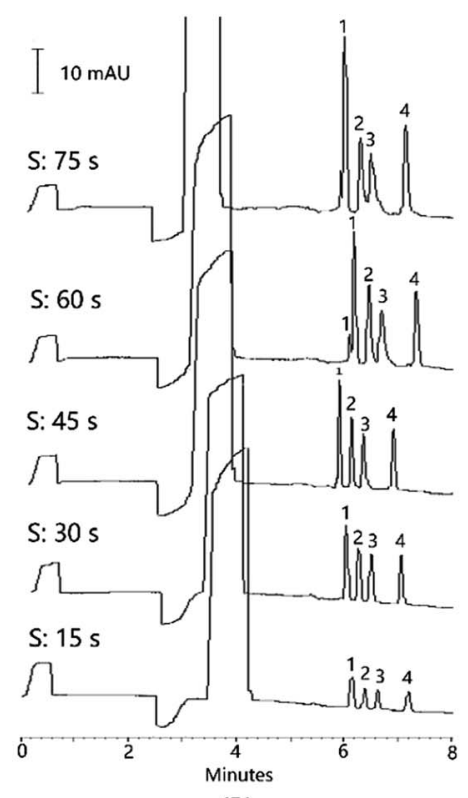

(B)

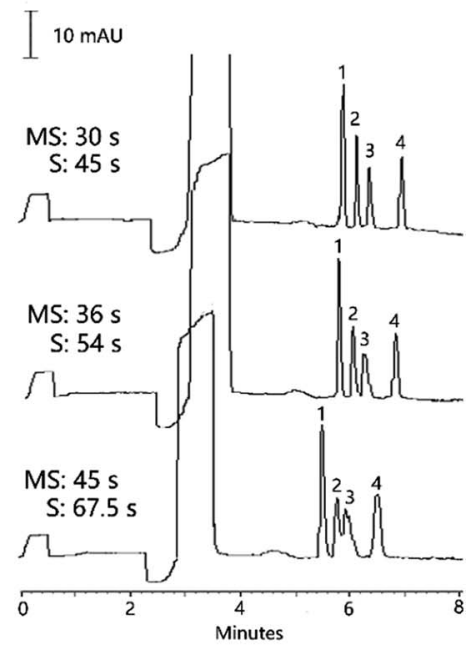

(C)

Fig. 3 The effect of the injection time of MS (A), S (B) and MS: S (C). Analyte concentration: $5 \mu \mathrm{g} \mathrm{mL}{ }^{-1}$ of the organic acids and IS each. The other conditions are the optimum conditions. Peak identification: 1-vanillic acid, 2-ferulic acid, 3-IS and 4-cinnamic acid. (MS: micellar solution; S: sample solution). 
Table 1 The quantitative parameters obtained for our method

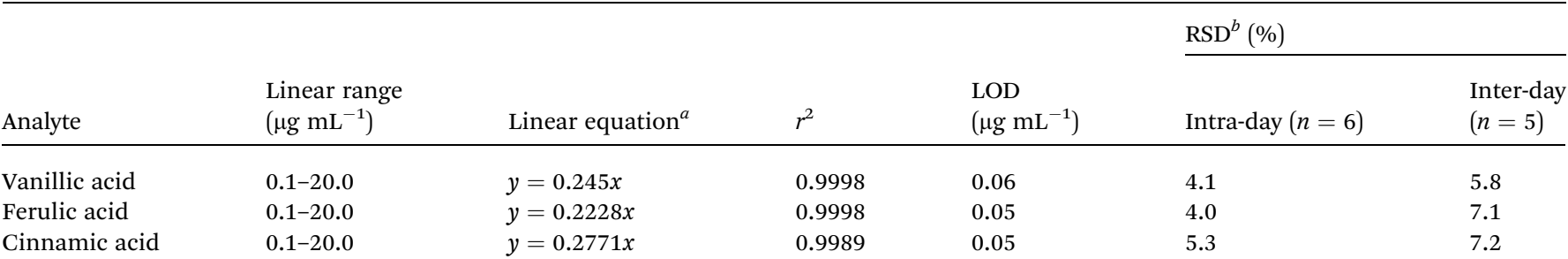

${ }^{a} y$ : The relative corrected peak area $=$ corrected peak area of analyte/corrected peak area of IS and $x$ : concentration. ${ }^{b}$ The concentration of each organic acid was $2.5 \mu \mathrm{g} \mathrm{mL}{ }^{-1}$. The other conditions were the optimum conditions.

range of 15 to $75 \mathrm{~s}$ was investigated. The peak heights for the three analytes increased with the extension of the injection time of the sample solution from 15 to 45 s. However, when the injection time was longer, the micellar solution was relatively insufficient, leading to a poor stacking efficiency for vanillic acid (Fig. 3B). Besides, a declines in both the resolution between ferulic acid and IS, and the separation efficiencies for all the compounds was observed when the injection time was

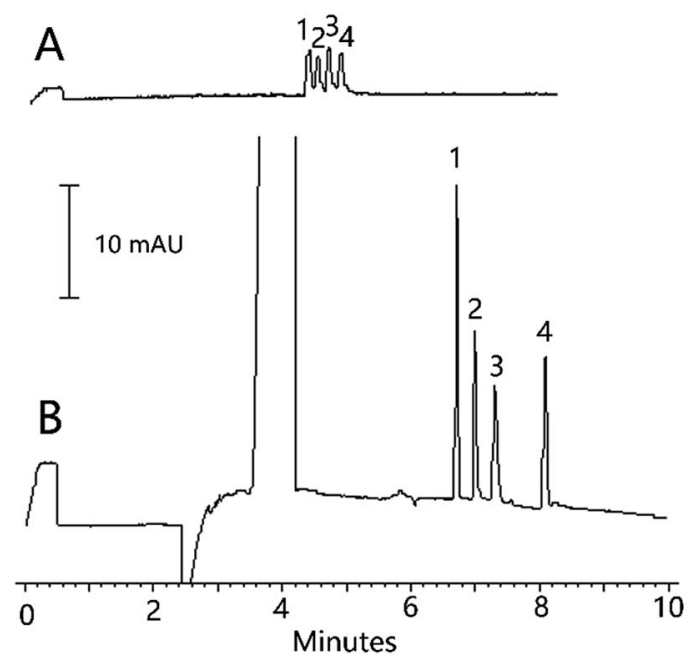

Fig. 4 A comparison of the electropherograms obtained using normal CZE (the three analytes and IS (25.0 $\mu \mathrm{g} \mathrm{mL}^{-1}$ each)) (A) and sweepingMSS-CZE (the three analytes and IS (5.0 $\mu \mathrm{g} \mathrm{mL}^{-1}$ each)) (B); detection wavelength: $215 \mathrm{~nm}$; peak identification: 1-vanillic acid, 2-ferulic acid, 3-IS and 4-cinnamic acid. increased from 45 to 75 (Table $\mathrm{S} 3 \dagger$ ). As a result, the injection time ratio of the micellar solution to sample solution was maintained at $2: 3$. When the injection time for both the micellar solution and sample solution were further increased simultaneously, both the resolution between the ferulic acid and IS and the separation efficiency were seriously decreased (Fig. 3C and Table $\mathrm{S} 4 \dagger$ ). As a result, the micellar solution and sample solution injections were selected at 0.5 psi for $30 \mathrm{~s}$ and $45 \mathrm{~s}$, respectively.

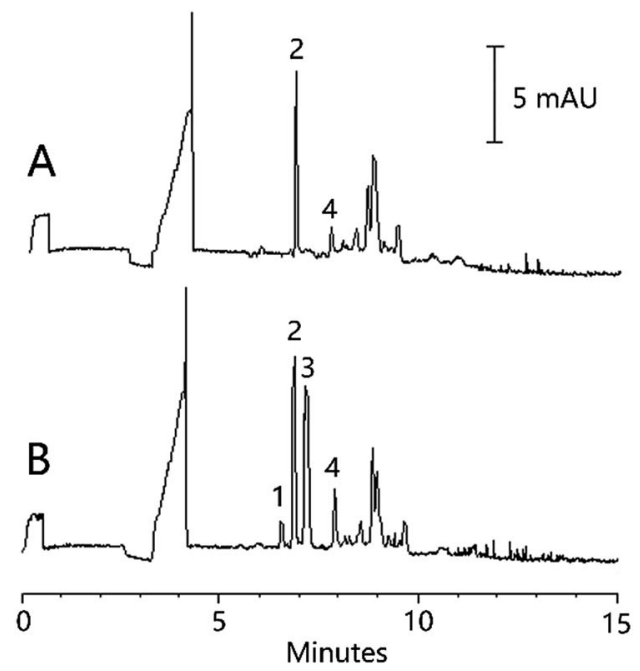

Fig. 5 The electropherograms obtained for the Angelica Sinensis sample (A) and the sample spiked with the analytes $\left(0.125 \mathrm{mg} \mathrm{g}^{-1}\right.$ each) (B); peak identification: 1-vanillic acid, 2-ferulic acid, 3-IS and 4-cinnamic acid.

Table 2 The results obtained for the determination of the analytes in Angelica sinensis and method recoveries ${ }^{a}$

\begin{tabular}{|c|c|c|c|c|c|}
\hline Ingredient & $\begin{array}{l}\text { Content } \\
\left(\mathrm{mg} \mathrm{g}^{-1}\right)\end{array}$ & $\begin{array}{l}\text { Spiked } \\
\left(\mathrm{mg} \mathrm{g}^{-1}\right)\end{array}$ & $\begin{array}{l}\text { Found } \\
\left(\mathrm{mg} \mathrm{g}^{-1}\right)\end{array}$ & $\begin{array}{l}\text { Recovery } \\
(\%)\end{array}$ & $\begin{array}{l}\operatorname{RSD}(\%) \\
(n=3)\end{array}$ \\
\hline Vanillic acid & ND & 0.125 & 0.118 & 94.4 & 7.5 \\
\hline \multirow[t]{2}{*}{ Ferulic acid } & \multirow[t]{2}{*}{0.74} & 0.125 & 0.861 & 96.8 & 4.5 \\
\hline & & 0.25 & 1.011 & 108.4 & 5.7 \\
\hline Cinnamic acid & 0.09 & 0.125 & 0.209 & 95.2 & 6.2 \\
\hline
\end{tabular}

${ }^{a} \mathrm{ND}$, not detected. 
Table 3 A comparison of the current method with the previously reported methods used for the analysis of organic acids

\begin{tabular}{|c|c|c|c|c|c|c|}
\hline Method & Sample & $\begin{array}{l}\text { Linearity }(\mu \mathrm{g} \\
\left.\mathrm{mL}^{-1} \text { or } \mu \mathrm{g} \mathrm{g}^{-1}\right)\end{array}$ & $\begin{array}{l}\operatorname{LOD}(\mu \mathrm{g} \\
\left.\mathrm{mL}^{-1} \text { or } \mu \mathrm{g} \mathrm{g}^{-1}\right)\end{array}$ & RSD (\%) & $\begin{array}{l}\text { Analysis time } \\
\text { (min) }\end{array}$ & Ref. \\
\hline HPLC & Rat plasma & $0.037-3.7$ & 0.012 & $<8.1$ & 25 & 6 \\
\hline HPLC & Aromatic plants & $0.09-7.21$ & 0.05 & $3.2-6.9$ & 110 & 9 \\
\hline MEKC & Tomato & $1-20$ & $0.8-3.8$ & $<4.4$ & 15 & 14 \\
\hline CZE & Brassica oleracea & $1-500$ & $1.1-2.3$ & $<3.6$ & 6.5 & 12 \\
\hline
\end{tabular}

\subsection{Quantitative analysis}

The calibration curve, limits of detection (LODs), correlation coefficient $\left(r^{2}\right)$ and repeatability were studied under the above optimized conditions for the analysis of the three organic acids. The results are summarized in Table 1 . The corrected peak area was defined as the ratio between the peak area and the migration time. A series of standard sample solutions containing each of the organic acids at nine concentration levels of 0.1 , $0.25,0.5,1.0,2.5,5.0,10.0,15.0$ and $20.0 \mu \mathrm{g} \mathrm{mL}{ }^{-1}$, as well as 5.0 $\mu \mathrm{g} \mathrm{mL}^{-1}$ IS each were prepared for obtaining the calibration curves. The calibration curves were obtained by plotting the mean relative corrected peak areas (corrected peak area of the analyte/corrected peak area of the IS) versus the concentrations of the individual analyte with three replicate measurements. As a result, a good linear relationship $\left(r^{2}>0.9989\right)$ was obtained for all the studied organic acids. LOD $(\mathrm{S} / \mathrm{N}=3)$ for vanillic acid, ferulic acid and cinnamic acid was $0.05,0.05$ and $0.06 \mu \mathrm{g} \mathrm{mL}^{-1}$, respectively. The intra- and inter-day repeatabilities expressed as relative standard deviations (RSDs) were studied using the $2.5 \mu \mathrm{g} \mathrm{mL}{ }^{-1}$ standard. The resulting intra-day $(n=6)$ and interday $(n=5)$ RSDs of the peak areas ranged from 4.1 to $5.3 \%$ and 5.8 to $7.2 \%$, respectively.

For the normal CZE analysis, the best separation was achieved in $150 \mathrm{mM} \mathrm{H}_{3} \mathrm{BO}_{3}(\mathrm{pH} 8.5)$ at a voltage of $20 \mathrm{kV}$ at $25^{\circ} \mathrm{C}$. The mixed standard solution containing the three analytes and IS $\left(25.0 \mu \mathrm{g} \mathrm{mL}^{-1}\right.$ each) was prepared in $150 \mathrm{mM} \mathrm{H}_{3} \mathrm{BO}_{3}(\mathrm{pH} \mathrm{8.5})$. Then, it was injected into the capillary at 0.5 psi for $3 \mathrm{~s}$. The electropherogram for the analysis of the organic acids by normal CZE is shown in Fig. 4A, and the result obtained by the current method with the sample solution containing the three analytes and IS $\left(5.0 \mu \mathrm{g} \mathrm{mL} \mathrm{m}^{-1}\right.$ each) is presented in Fig. 4B. When compared with those for the normal CZE injection procedure, 77, 52 and 42-fold sensitivity enhancements for vanillic acid, ferulic acid and cinnamic acid, respectively were achieved using the current method.

\subsection{Real sample analysis}

The developed stacking preconcentration method was used for the analysis of three organic acids in the commonly used TCM, Angelica sinensis. The results are shown in Table 2. The Angelica sinensis sample was found to contain ferulic acid and cinnamic acid at $0.74 \mathrm{mg} \mathrm{g}^{-1}$ and $0.09 \mathrm{mg} \mathrm{g}^{-1}$, respectively. No vanillic acid was found in the sample. The determined recoveries of the analytes for the method were in the range of $94.4 \%$ to $108.4 \%$ with RSDs between $4.5 \%$ and $7.5 \%$. Fig. 5 shows the typical chromatograms obtained for the Angelica sinensis sample before and after being spiked with each of the organic acids.

\subsection{A comparison with other analytical techniques}

The performance of the current method based on sweepingMSS-CZE used for the determination of organic acids was compared with that of the relevant methods previously reported in the literature. As listed in Table 3, the two-step stacking method is more sensitive than one-step stacking ${ }^{\mathbf{1 3}}$ or no stacking methods. ${ }^{12,14}$ In addition, the obtained LOD values were comparable to those of the HPLC methods reported by other authors with DAD detection. ${ }^{6,7,9}$ However, the separation time of CE is much shorter than that of HPLC.

\section{Conclusions}

The sweeping-MSS method was developed for the determination of three organic acids found in Angelica sinensis. The technique used for the analysis of anions was successfully applied in the TCM sample. In comparison with that of normal CZE, the detection sensitivity for the organic acids was enhanced 42-77-fold. The present method is suitable and favourable for the analysis of organic acids on account of its cost effectiveness, rapidity, simplicity and sensitivity.

\section{Conflicts of interest}

There are no conflicts of interest to declare.

\section{Acknowledgements}

The authors acknowledge the financial support of the Natural Science Foundation of Hebei Province (B2016204146) and the Science and Technology Foundation of Hebei Agricultural University (LG201607).

\section{References}

1 W. L. Wei, R. Zeng, C. M. Gu, Y. Qu and L. F. Huang, J. Ethnopharmacol., 2016, 190, 116-141.

2 M. Jin, K. Zhao, Q. Huang, C. Xu and P. Shang, Carbohydr. Polym., 2012, 89, 713-722. 
3 D. Zhou, N. Li, Y. Zhang, C. Yan, K. Jiao, Y. Sun, H. Ni, B. Lin and Y. Hou, RSC Adv., 2016, 6, 97302-97312.

4 K. Zhang, G. Yan, A. Zhang, H. Sun and X. Wang, RSC Adv., 2017, 7, 28876-28888.

5 G. H. Lu, K. Chan, K. Leung, C. L. Chan, Z. Z. Zhao and Z. H. Jiang, J. Chromatogr., A, 2005, 1068, 209-219.

6 J. Qi, X. Jin, L. Huang and Q. Ping, Biomed. Chromatogr., 2007, 21, 816-822.

7 A. Skendi, M. Irakli and P. Chatzopoulou, Journal of Applied Research on Medicinal and Aromatic Plants, 2017, 6, 62-69.

8 M.-A. Jabri, N. Hajji, D. Wannes, H. Tounsi, M. Jridi, A. Abdellaoui, M. Nasri, L. Marzouki and H. Sebai, RSC Adv., 2017, 7, 53472-53480.

9 C. Proestos, M. Kapsokefalou and M. Komaitis, J. Food Qual., 2008, 31, 13.

10 Q. Xue, H. Fan, K. Li, L. Yang, L. Sun and Y. Liu, RSC Adv., 2017, 7, 51151-51161.

11 G. Ding, Y. Wang, A. Liu, Y. Hou, T. Zhang, G. Bai and C. Liu, RSC Adv., 2017, 7, 22034-22044.

12 I. S. L. Lee, M. C. Boyce and M. C. Breadmore, Food Chem., 2011, 127, 797-801.

13 C. A. Ballus, A. D. Meinhart, R. G. de Oliveira and H. T. Godoy, Food Res. Int., 2012, 45, 136-144.

14 R. Martí, M. Valcárcel, J. M. Herrero-Martínez, J. CebollaCornejo and S. Roselló, Food Chem., 2017, 221, 439-446.

15 M. C. Breadmore, A. Wuethrich, F. Li, S. C. Phung, U. Kalsoom, J. M. Cabot, M. Tehranirokh, A. I. Shallan,
A. S. Abdul Keyon, H. H. See, M. Dawod and J. P. Quirino, Electrophoresis, 2017, 38, 33-59.

16 X. Hou, X. Zhang and Y. Lu, Anal. Methods, 2017, 9, 10-17. 17 J. P. Quirino, J. Chromatogr., A, 2009, 1216, 294-299.

18 X. Yang, S. Zhang, C. Wang and Z. Wang, Chin. J. Anal. Chem., 2013, 41, 1939-1946.

19 J. P. Quirino, J. Chromatogr., A, 2010, 1217, 7776-7780.

20 X. Yang, X. Cheng, Y. Lin, Z. Tan, L. Xie and M. M. F. Choi, J. Chromatogr., A, 2014, 1325, 227-233.

21 X. Yang, S. Zhang, J. Wang, C. Wang and Z. Wang, Anal. Chim. Acta, 2014, 814, 63-68.

22 J. P. Quirino and A. T. Aranas, Anal. Chim. Acta, 2012, 733, 84-89.

23 C. Kukusamude, S. Srijaranai, M. Kato and J. P. Quirino, J. Chromatogr., A, 2014, 1351, 110-114.

24 L. Y. Thang, H. H. See and J. P. Quirino, Talanta, 2016, 161, 165-169.

25 A. Wuethrich, P. R. Haddad and J. P. Quirino, Electrophoresis, 2016, 37, 1139-1142.

26 W. Grochocki, M. J. Markuszewski and J. P. Quirino, J. Chromatogr., A, 2015, 1424, 111-117.

27 W. Grochocki, M. J. Markuszewski and J. P. Quirino, J. Chromatogr., A, 2016, 1442, 140-143.

28 J. P. Quirino and A. M. Guidote Jr, J. Chromatogr., A, 2011, 1218, 1004-1010.

29 J. P. Quirino, P. Anres, J. Sirieix-Plénet, N. Delaunay and P. Gareil, J. Chromatogr., A, 2011, 1218, 5718-5724. 